$\mathrm{R} / 96 / 60$

December, 1996

Revised, May 15 and July 10

\title{
Superstring dualities and p-brane bound states
}

\author{
M.S. Costa and G. Papadopoulos \\ D.A.M.T.P \\ University of Cambridge \\ Silver Street \\ Cambridge CB3 9EW
}

\begin{abstract}
We show that the M-theory/IIA and IIA/IIB superstring dualities together with the diffeomorphism invariance of the underlying theories require the presence of certain p-brane bound states in IIA and IIB superstring theories preserving $1 / 2$ of the spacetime supersymmetry. We then confirm the existence of IIA and IIB supergravity solutions having the appropriate p-brane bound states interpretation.
\end{abstract}




\section{Introduction}

Much evidence have been gathered in the past year to support the conjecture that the strong coupling limit of IIA superstring theory is an 11-dimensional supersymmetric theory called M-theory [1, 2]. More precisely, the IIA superstring theory is the compactification of M-theory on a $S^{1}$ with the IIA string coupling constant $\lambda$ related to the compactification radius $R$ as $\lambda=R^{2 / 3}$. This interpretation of the IIA superstring theory requires the presence of non-perturbative states in IIA some of which should carry Ramond-Ramond (RR) charges. The first indication that such states were present in IIA superstrings was the existence of solutions of $\mathrm{D}=10$ IIA supergravity carrying the appropriate charges $[3,4]$, subsequently this was confirmed within IIA superstring theory by identifying the IIA $\mathrm{D}$-p-branes for $\mathrm{p}=0,2,4,6,8$ as the carriers of $\mathrm{RR}$ charges [5]. The conjectured $\mathrm{D}=11$ Kaluza-Klein (KK) origin of IIA superstrings further requires that all IIA p-branes should have a $\mathrm{D}=11$ interpretation [1]. It turns out that this is the case with the IIA membrane and 5-brane being the 'direct' reduction of M-branes, and the IIA string and 4-brane being the 'double' reduction of M-branes. The rest of the IIA p-branes, the D-0-branes and D-6-branes ${ }^{\star}$, are interpreted as the KK modes and the KK monopoles of the reduction from $\mathrm{D}=11$ to $\mathrm{D}=10$, respectively. In addition, the interpretation of the IIA string and 4-brane as the double reduction of M-branes requires the existence of BPS-saturated 0-brane/p-brane bound states for $\mathrm{p}=1,4$, preserving precisely $1 / 4$ of the spacetime supersymmetry $[6,7]$. The corresponding supergravity solutions were given in [7] thus providing more evidence for M-theory/IIA duality.

Another novel equivalence is the T-duality of IIA and IIB superstring theories. The IIA superstring theory compactified on a circle of radius $R_{A}$ is equivalent to the IIB superstring theory compactified on a circle of radius $R_{B}$ with $R_{B}=\alpha^{\prime} / R_{A}$ where $\alpha^{\prime}$ is the string tension $[8,9]$. T-duality is a perturbative superstring symme-

\footnotetext{
* The IIA superstring theory also has an 8-brane but no direct $\mathrm{D}=11$ interpretation has been found for it as yet.
} 
try and therefore this equivalence between IIA and IIB superstrings can be verified to all orders in string perturbation theory. As in the M-theory/IIA duality, the IIA p-branes transform under IIA/IIB T-duality to the IIB p-branes and vice-versa. In particular, IIA D-p-branes for $\mathrm{p}=0,2,4,6,8$ transform under T-duality either to the IIB D-(p-1)-branes or to the IIB D-(p+1)-branes depending on whether the Tduality operation is taken along a worldvolume or a transverse direction of the IIA D-p-branes, respectively. Similarly, the IIB D-p-branes for $\mathrm{p}=1,3,5,7$, transform under T-duality either to the IIA D-(p-1)-branes or to the IIA D- $(\mathrm{p}+1)$-branes ${ }^{\dagger}$. Furthermore in the effective theory, the fundamental IIA (IIB) string and the solitonic IIA (IIB) 5-brane transform under T-duality along a transverse direction to the IIB (IIA) fundamental string and to the IIB (IIA) KK monopole, respectively. Moreover, the fundamental IIA (IIB) string and the solitonic IIA (IIB) 5-brane transform under T-duality along a worldvolume direction to the IIB (IIA) plane wave and to the IIB (IIA) solitonic 5-brane, respectively.

The KK reduction from M-theory to IIA superstring theory and the T-duality operation from IIA (IIB) to IIB (IIA) require a choice of a spacetime direction. But in diffeomorphic invariant theories, like M-theory, IIA and IIB superstrings, there is no such prefered direction and therefore the M-theory/IIA and IIA/IIB dualities should be independent of this choice. For example, the M-theory should reduce to the IIA superstring along any spatial $D=11$ direction. However as we have mentioned above, the interpretation of p-branes after a reduction or a $\mathrm{T}$ duality transformation depends on whether these operations are performed along one of their worldvolume or along one of their transverse directions. As we shall see, a reduction or a T-duality transformation along a generic direction requires the inclusion of new BPS-saturated states in the spectrum of IIA and IIB superstrings. These BPS-saturated states are below threshold and have the interpretation of pbrane bound states preserving $1 / 2$ of the spacetime supersymmetry; we shall list these p-brane bound states in section 2 . The presence of these p-brane bound states

\footnotetext{
$\dagger$ See [11] for the T-duality transformation on the D-branes from the superstring point of view.
} 
in IIA and IIB superstring theory is required by the M-theory/IIA and IIA/IIB dualities together with the diffeomorphism invariance of the underlying theories. This association of p-brane bound states to the M-theory/IIA and IIA/IIB dualities is similar to the association of KK modes to the KK reduction. Therefore, the existence of these p-brane bound states in IIA and IIB superstrings is necessary for the consistency of these duality conjectures. Some of the p-brane bound states that we shall consider in this paper have already been investigated either in the context of D-branes $[10,11,12,13,14]$ or from the effective theory point of view $[15,16,17,18]$. They are also required for the consistency of other superstring duality conjectures $[19,20]$.

We shall describe the above p-brane bound states from the macroscopic point of view. However in certain cases we shall also comment on their microscopic properties. The evidence that we shall present for the existence of these p-brane bound states and for their interpretation is derived from consideration of the solutions of the associated effective supergravity theories with the analogous interpretation. To derive the solutions in IIA and IIB supergravity with the desirable interpretation, we shall start from the solution of $D=11$ supergravity with the interpretation of a membrane/fivebrane bound state preserving $1 / 2$ of the spacetime supersymmetry [15]. This solution will be reduced to solutions of IIA supergravity using the 'standard' reduction along either a worldvolume or a transverse direction of the configuration $[16,17]$. The reduced solutions have a IIA interpretation as bound states of two IIA p-branes preserving $1 / 2$ of the spacetime supersymmetry. Then starting from these IIA solutions and using the T-duality rules [21], we shall construct 'standard' T-duality chains as in [7], i.e. T-duality transformations along worldvolume or transverse directions of these configurations, to find most of the required p-brane bound state solutions in IIA and IIB supergravity theories. These p-brane bound states will also be derived using KK reductions and T-duality transformations from the p-brane solutions of $\mathrm{D}=11$, IIA and IIB supergravities but this time these operations will be taken along a generic direction in spacetime. In this way, we shall establish that the origin of these bound states is due to 
the M-theory/IIA and IIA/IIB dualities and the diffeomorphism invariance of the underlying theories.

The organization of this paper is as follows: In section two, we shall present the bound states of IIA and IIB superstring theories expected from the reduction of M-theory to IIA and from the IIA/IIB T-duality. In section three, we shall give the IIA supergravity solutions with the interpretation of p-brane bound states that are expected from the reduction of M-theory to IIA superstring. In section four, we shall give the IIA and IIB supergravity solutions with the interpretation of p-brane bound states that are expected from the IIA/IIB duality. In section five, we shall present an M-theory interpretation of some of these solutions, and in section six we shall give our conclusions.

\section{2. p-brane bound states}

The reduction and T-duality operations do not commute with spacetime diffeo-

morphisms. Because of this there are many ways to choose a direction in spacetime to perform a reduction or a T-duality transformation. However, as in [18], here we shall consider only the following two cases: (i) In the first case, we shall perform the reduction and the T-duality transformation along a spatial spacetime direction which can be decomposed as a linear combination of a spatial worldvolume and a transverse direction of a p-brane, i.e. this direction intersects the p-brane at an angle $\alpha$. We shall denote the corresponding operations with $R_{\alpha}$ and $T_{\alpha}$, and we shall refer to them as reduction at an angle and T-duality at an angle, respectively. For $\alpha=0$ or $\alpha=\pi / 2, R_{\alpha}$ and $T_{\alpha}$ become the standard reduction and T-duality transformations along a worldvolume or a transverse directions of the p-brane, respectively. Therefore $R_{\alpha}\left(T_{\alpha}\right)$ is a linear combination of a standard reduction (T-duality transformation) along a worldvolume direction and a standard reduction (T-duality transformation) along a transverse direction of a p-brane. (ii) In the second case, we shall perform the reduction and the T-duality transformation along a direction which is transverse to a p-brane and with the p-brane moving with 
relativistic velocity $v$ in this direction, i.e. the p-brane is 'boosted' in this direction. We shall denote the corresponding operations with $R_{v}$ and $T_{v}$, and we shall refer to them as reduction along a boost and T-duality along a boost, respectively. In what follows, we shall use $R$ and $T$ to denote the 'standard' reduction and the 'standard' T-duality transformation along either a worldvolume or a transverse direction of a p-brane. We shall also use, when it is necessary in order to avoid ambiguities, the subscripts $M, A$ and $B$ to denote the BPS states of the corresponding theories, and the subscripts $F, D$ and $S$ to denote the fundamental, Dirichlet and solitonic p-branes, respectively.

As we have already mentioned in the introduction, reducing the M-branes along one of their worldvolume directions (double reduction) as

$$
\begin{aligned}
& 2_{M} \stackrel{R}{\longrightarrow} 1_{F} \\
& 5_{M} \stackrel{R}{\longrightarrow} 4_{D}
\end{aligned}
$$

yields the IIA fundamental string and D-4-brane, while reducing them along one of their transverse directions (direct reduction) as

$$
\begin{aligned}
& 2_{M} \stackrel{R}{\longrightarrow} 2_{D} \\
& 5_{M} \stackrel{R}{\longrightarrow} 5_{S},
\end{aligned}
$$

yields the IIA D-2-brane and the solitonic 5-brane. Similarly, the reductions of the (purely gravitational) $\mathrm{D}=11$ plane wave and the $\mathrm{D}=11 \mathrm{KK}$ monopole to $\mathrm{D}=10$ are

$$
\begin{aligned}
& 0_{w} \stackrel{R}{\longrightarrow} 0_{w} \\
& 0_{w} \stackrel{R}{\longrightarrow} 0_{D},
\end{aligned}
$$

and

$$
\begin{aligned}
& 0_{m} \stackrel{R}{\longrightarrow} 0_{m} \\
& 0_{m} \stackrel{R}{\longrightarrow} 6_{D},
\end{aligned}
$$

respectively, where $0_{w}$ denotes the plane wave and $0_{m}$ denotes the KK monopole. 
Now since $R_{\alpha}$ is equivalent to a simultaneous double and direct reduction, reducing the M-p-branes at an angle leads to IIA (p-1)-brane/p-brane bound states. It is convenient to use the notation $(r \mid p, q)$ to denote a solution representing an r-brane intersection of a p-brane with a q-brane; in the special case where $p=r$, $(p \mid p, q)$ denotes a $\mathrm{p}$-brane within a q-brane representing a bound state of a p-brane with a q-brane. In this notation, the reduction of M-branes at an angle is described as

$$
\begin{aligned}
& 2_{M} \stackrel{R_{\alpha}}{\longrightarrow}(1 \mid 1,2)_{A} \\
& 5_{M} \stackrel{R_{\alpha}}{\longrightarrow}(4 \mid 4,5)_{A} .
\end{aligned}
$$

Similarly, the reduction of the $\mathrm{D}=11$ plane wave and the $\mathrm{D}=11 \mathrm{KK}$ monopole at an angle leads to the IIA bound states

$$
\begin{aligned}
& 0_{w} \stackrel{R_{\alpha}}{\longrightarrow}\left(0_{w} \mid 0\right)_{A} \\
& 0_{m} \stackrel{R_{\alpha}}{\longrightarrow}\left(0_{m} \mid 6\right)_{A},
\end{aligned}
$$

where $\left(0_{w} \mid 0\right)_{A}$ denotes a bound state of a plane wave with a 0 -brane, and $\left(0_{m} \mid 6\right)_{A}$ denotes the bound state of a KK monopole with a 6 -brane. The $\left(0_{w} \mid 0\right)_{A}$ bound state is a 'boosted' 0-brane.

Next, the reduction of the M-branes, the $\mathrm{D}=11$ plane wave and the $\mathrm{D}=11 \mathrm{KK}$ monopole along a boost leads to IIA bound states that always involve the IIA D-0brane. This is because from the $\mathrm{D}=10$ perspective the (quantised) momentum in the compactifying direction becomes the mass of the KK modes of the compactification. But as we have mentioned in the introduction, these are identified with the IIA D-0-branes. So this observation together with the fact that we reduce along a transverse direction of the configurations lead to the following IIA p-brane bound states:

$$
\begin{aligned}
& 2_{M} \stackrel{R_{v}}{\longrightarrow}(0 \mid 0,2)_{A} \\
& 5_{M} \stackrel{R_{v}}{\longrightarrow}(0 \mid 0,5)_{A} \\
& 0_{w} \stackrel{R_{v}}{\longrightarrow}\left(0 \mid 0_{w}\right)_{A} \\
& 0_{m} \stackrel{R_{v}}{\longrightarrow}(0 \mid 0,6)_{A} .
\end{aligned}
$$


We remark that it has been argued in [11] that there is not a IIA BPS D-0-brane/D6 -brane bound state. We shall return to this point when we discuss the $(0 \mid 0,6)_{A}$ solution of IIA supergravity in the next section. As in (2.6), the $\left(0_{w} \mid 0\right)_{A}$ bound state is a 'boosted' 0-brane.

We now turn to the p-brane bound states required by the IIA/IIB superstring duality. As we have already mentioned T-duality transforms a (IIA or IIB) D$(p+1)$-brane either as

$$
(p+1)_{D} \stackrel{T}{\rightarrow} p_{D}
$$

or as

$$
(p+1)_{D} \stackrel{T}{\longrightarrow}(p+2)_{D}
$$

depending on whether the T-duality transformation is performed along a worldvolume or a transverse direction of the $\mathrm{D}$ - $(\mathrm{p}+1)$-brane, respectively, where the p-brane and the $(\mathrm{p}+2)$-brane are (IIB or IIA) D-branes. Similarly, the IIA or IIB fundamental string, solitonic 5-brane, plane wave and KK monopole transform under T-duality as follows:

$$
\begin{array}{ll}
1_{F} \stackrel{T}{\longleftrightarrow} 1_{F}, & 1_{F} \stackrel{T}{\longleftrightarrow} 0_{w} \\
5_{S} \stackrel{T}{\longleftrightarrow} 5_{S}, & 5_{S} \stackrel{T}{\longleftrightarrow} 0_{m} \\
0_{w} \stackrel{T}{\longleftrightarrow} 0_{w}, & 0_{m} \stackrel{T}{\longleftrightarrow} 0_{m} .
\end{array}
$$

We remark that the transformation $1_{F} \leftrightarrow 0_{w}$ is the usual exchange between (IIA or IIB) winding modes and (IIB or IIA) momentum modes of the fundamental string under T-duality.

Now since $T_{\alpha}$ is a linear combination of a T-duality transformation along a worldvolume direction and a T-duality transformation along a transverse direction of a p-brane, it is clear from (2.8) and (2.9) that acting with a T-duality at an angle on a (IIA or IIB) D-(p+1)-brane will lead to BPS-saturated (IIB or IIA) 
D-p-brane/D-(p+2)-brane bound states preserving $1 / 2$ of the spacetime supersymmetry, i.e.

$$
(p+1)_{D} \stackrel{T_{\alpha}}{\longrightarrow}\left(p \mid p_{D},(p+2)_{D}\right)
$$

The $\left(p \mid p_{D},(p+2)_{D}\right)$, for $p=0,2,4,6$, are bound states of IIA superstring theory and $\left(p \mid p_{D},(p+2)_{D}\right)$, for $p=1,3,5$, are bound states of IIB superstring theory. The existence of the bound states $\left(p \mid p_{D},(p+2)_{D}\right)$ preserving $1 / 2$ of the spacetime supersymmetry is also expected from D-brane considerations. Similarly, applying T-duality at an angle to the (IIA or IIB) fundamental string, solitonic 5-brane, plane wave and KK monopole leads to the following bound states:

$$
\begin{aligned}
& 1_{F} \stackrel{T_{\alpha}}{\longrightarrow}\left(0_{w} \mid 1_{F}\right) \\
& 5_{S} \stackrel{T_{\alpha}}{\longrightarrow}\left(0_{m} \mid 5_{S}\right) \\
& 0_{w} \stackrel{T_{\alpha}}{\longrightarrow}\left(0_{w} \mid 1_{F}\right) \\
& 0_{m} \stackrel{T_{\alpha}}{\longrightarrow}\left(0_{m} \mid 5_{S}\right) .
\end{aligned}
$$

The bound state $\left(0_{w} \mid 1_{F}\right)$ is simply a boosted fundamental string.

Next, the p-brane bound states expected from applying T-duality along a boost to the IIA and IIB p-branes will always involve the fundamental (IIA or IIB) string. This is because the (quantised) momentum modes along the direction of the Tduality transformation become in the dual picture the winding modes of the (IIA or IIB) fundamental string. This observation together with the fact that the direction of the boost is transverse to the objects lead to the following bound states involving the (IIA or IIB) D-p-branes:

$$
p_{D} \stackrel{T_{v}}{\longrightarrow}\left(1 \mid 1_{F},(p+1)_{D}\right)
$$

where $p=0, \ldots, 7$. An alternative explanation for the presence of the fundamental string/D- $(\mathrm{p}+1)$-brane bound state can be found from consideration of the D-pbrane effective action. In this case, T-duality turns velocity in a transverse direction 
of a p-brane to an electromagnetic field in the dual picture. But the flux of the electomagnetic field is related to the tension of the fundamental string [22], so in the dual picture one finds a fundamental string/D- $(\mathrm{p}+1)$-bound state. Similarly, the expected bound states from applying T-duality along a boost to the (IIA or IIB) fundamental string, solitonic 5-brane, plane wave and KK monopole are

$$
\begin{aligned}
& 1_{F} \stackrel{T_{v}}{\longrightarrow} 1_{F} \\
& 5_{S} \stackrel{T_{v}}{\longrightarrow}\left(0_{m} \mid 1_{F}\right) \\
& 0_{w} \stackrel{T_{v}}{\longrightarrow}\left(0_{w} \mid 1_{F}\right) \\
& 0_{m} \stackrel{T_{v}}{\longrightarrow}\left(1 \mid 1_{F}, 5_{S}\right) .
\end{aligned}
$$

We remark that, as we shall see in section 4, the supergravity solution associated with the $\left(1 \mid 1_{F}, 5_{S}\right)$ bound state has similar qualitative features as the solution that is interpreted as a $(0 \mid 0,6)_{A}$ bound state.

So far we have listed the bound states that we expect to find in IIA and IIB superstring theories due to the M-theory/IIA and IIA/IIB dualities and to the diffeomorphism invariance of the underlying theories. Evidence for the existence of all these bound states will be given in the next two sections by finding the solutions of the corresponding supergravity theories with the appropriate interpretation. We have been able to carry out this computation for all the required p-brane bound states. The resulting IIA and IIB p-brane bound state solutions will be expressed in the string frame. All of them have a parameter $\alpha$ which interpolates between the two constituent objects that form the bound states. 


\section{M-theory/IIA duality and bound states}

As we have explained in the previous section, M-theory/IIA duality requires the existence of the bound states $(1 \mid 1,2)_{A}$ and $(4 \mid 4,5)_{A}$ in IIA superstring theory preserving $1 / 2$ of the spacetime supersymmetry. The corresponding supergravity solutions can be obtained starting from the $\mathrm{D}=11$ supergravity solution $(2 \mid 2,5)_{M}$ [15],

$$
\begin{aligned}
d s^{2} & =(H \tilde{H})^{\frac{1}{3}}\left[H^{-1} d s^{2}\left(\mathbb{M}^{3}\right)+\tilde{H}^{-1} d s^{2}\left(\mathbb{E}^{3}\right)+d s^{2}\left(\mathbb{E}^{5}\right)\right] \\
G_{4} & =\sin \alpha \epsilon\left(\mathbb{M}^{3}\right) \wedge d H^{-1}-\cos \alpha \star d H+\tan \alpha \epsilon\left(\mathbb{E}^{3}\right) \wedge d \tilde{H}^{-1},
\end{aligned}
$$

preserving $1 / 2$ of the spacetime supersymmetry, where $H$ is a harmonic function on $\mathbb{E}^{5}, \tilde{H}=\sin ^{2} \alpha+\cos ^{2} \alpha H$, star is the Hodge star in $\mathbb{E}^{5}$ and $\alpha \in[0, \pi / 2]$ is an angle parameter of the solution; this solution interpolates between the membrane and fivebrane solutions of $\mathrm{D}=11$ supergravity theory by adjusting the parameter $\alpha$. In what follows, we shall take the harmonic function $H \sim 1$ at the transverse spatial infinity. The IIA $\left(1 \mid 1_{F}, 2\right)_{A}$ and $\left(4 \mid 4,5_{S}\right)_{A}$ solutions can be derived from the following T-duality chains:

$$
(2 \mid 2,5)_{M} \stackrel{R}{\rightarrow}\left(1 \mid 1_{F}, 4\right)_{A} \stackrel{T}{\rightarrow}\left(1 \mid 1_{F}, 3\right)_{B} \stackrel{T}{\rightarrow}\left(1 \mid 1_{F}, 2\right)_{A}
$$

and

$$
(2 \mid 2,5)_{M} \stackrel{R}{\rightarrow}\left(2 \mid 2,5_{S}\right)_{A} \stackrel{T}{\rightarrow}\left(3 \mid 3,5_{S}\right)_{B} \stackrel{T}{\rightarrow}\left(4 \mid 4,5_{S}\right)_{A} .
$$

It should also be possible to construct the $(1 \mid 1,2)_{A}$ and $(4 \mid 4,5)_{A}$ solutions of IIA supergravity by KK reduction of the M-brane solutions at an angle. This computation was done in [18] by first rotating the M-brane solutions and then reducing them to $\mathrm{D}=10$. Here we shall repeat this computation and relate the result to that of the T-duality chains (3.2) and (3.3). The M-brane solutions 
$[23,24]$ are

$$
\begin{aligned}
& d s^{2}=H^{(p+1) / 9}\left[H^{-1} d s^{2}\left(\mathbb{M}^{p+1}\right)+d s^{2}\left(\mathbb{E}^{10-p}\right)\right] \\
& G_{4}= \begin{cases}\epsilon\left(\mathbb{M}^{3}\right) \wedge d H^{-1}, & p=2 \\
-\star d H, & p=5\end{cases}
\end{aligned}
$$

where $\mathbb{E}^{n}$ is the Euclidean space of dimension $n, \mathbb{M}^{n}$ is the Minkowski space of dimension $n, H$ is a harmonic function on $\mathbb{E}^{10-p}, \epsilon(\mathcal{M})$ is the volume form of a manifold $\mathcal{M}$, and the star is the Hodge star in $\mathbb{E}^{5}$. We also write for later use the (purely gravitational) $\mathrm{D}=11$ plane wave solution

$$
d s^{2}=-d t^{2}+d \rho^{2}+(H-1)(d t+d \rho)^{2}+d s^{2}\left(\mathbb{E}^{9}\right)
$$

where $H$ is a harmonic function on $\mathbb{E}^{9}$, and the $\mathrm{D}=11 \mathrm{KK}$ monopole solution

$$
d s^{2}=d s^{2}\left(\mathbb{M}^{7}\right)+d s^{2}(G / H)
$$

where $d s^{2}(G / H)=H^{-1}(d \rho+\omega)^{2}+H d s^{2}\left(\mathbb{E}^{3}\right)$ is the Gibbons-Hawking metric [25], $H$ is a harmonic function on $\mathbb{E}^{3}$ and $d \omega={ }^{*} d H$. Both the plane wave $[26,27]$ and the KK monopole preserve $1 / 2$ of the spacetime supersymmetry; for the latter case this follows from the fact that $d s^{2}(G / H)$ is hyper-Kähler. To choose the compactifying direction $u_{11}$ in $\mathrm{D}=11$, we use a $\mathrm{D}=11$ diffeomorphism that mixes the spatial worldvolume and the transverse space coordinates of the M-branes. An example of such diffeomorphism is

$$
\left(\begin{array}{l}
u_{10} \\
u_{11}
\end{array}\right)=A\left(\begin{array}{l}
\rho \\
y
\end{array}\right)
$$

where $\rho$ is the coordinate along a spatial worldvolume direction, $y$ is the coordinate along a transverse direction and $A$ is a real invertible $2 \times 2$ matrix. Let us choose

$$
A=\left(\begin{array}{cc}
\cos \alpha & -\sin \alpha \\
\sin \alpha & \cos \alpha
\end{array}\right)
$$

where $\alpha$ is the angle that appears as a parameter in the $(2 \mid 2,5)_{M}$ solution above. The solutions of IIA supergravity that are constructed by reducing the M-branes 
along $u_{11}[18]$ are identical to the solutions found by the duality chains (3.2) and (3.3) applied to the $(2 \mid 2,5)_{M}$ solution. This confirms that the presence of $(1 \mid 1,2)_{A}$ and $(4 \mid 4,5)_{A}$ solutions is a consequence of the M-theory/IIA duality and the diffeomorphism invariance of M-theory. Conversely, the existence of these solutions serve as further evidence for the M-theory/IIA duality.

As we have already mentioned in section 2 , reducing the $\mathrm{D}=11$ plane wave and the KK monopole at an angle leads to the bound states $\left(0_{w} \mid 0\right)_{A}$ and $\left(0_{m} \mid 6\right)_{A}$, respectively. The $\left(0_{w} \mid 0\right)_{A}$ solution may be found by extending the T-duality chain $(3.2)$ as

$$
\left(1 \mid 1_{F}, 2\right)_{A} \stackrel{T}{\rightarrow}\left(1 \mid 1_{F}, 1_{D}\right)_{B} \stackrel{T}{\rightarrow}\left(0_{w} \mid 0\right)_{A},
$$

where $\left(0_{w} \mid 0\right)_{A}$ is

$$
\begin{aligned}
d s^{2} & =\tilde{H}^{\frac{1}{2}}\left[-H^{-1} d t^{2}+H \tilde{H}^{-1}\left(d x+\sin \alpha(H-1) H^{-1} d t\right)^{2}+d s^{2}\left(\mathbb{E}^{8}\right)\right] \\
e^{\phi} & =\tilde{H}^{\frac{3}{4}} \\
F_{2} & =\cos ^{-1} \alpha d t \wedge d \tilde{H}^{-1}+\tan \alpha d x \wedge d \tilde{H}^{-1} .
\end{aligned}
$$

This solution is interpreted as a 'boosted' 0-brane and is diffeomorphic (up to a rescalling of its mass, i.e. a rescalling of $(H-1))$ to the 0-brane solution of IIA supergravity. Note that varying the parameter $\alpha$, the solution (3.10) interpolates between the IIA plane wave solution $(\alpha=\pi / 2)$ and the IIA D-0-brane $(\alpha=0)$. Similarly, the $\left(0_{m} \mid 6\right)_{A}$ solution can be obtained by extending the T-duality chain (3.3) as

$$
\left(4 \mid 4,5_{S}\right)_{A} \stackrel{T}{\rightarrow}\left(5 \mid 5_{D}, 5_{S}\right)_{B} \stackrel{T}{\rightarrow}\left(0_{m} \mid 6\right)_{A} ;
$$

the $\left(0_{m} \mid 6\right)_{A}$ solution is

$$
\begin{aligned}
d s^{2} & =(\tilde{H} H)^{\frac{1}{2}}\left[H^{-1} d s^{2}\left(\mathbb{M}^{6}\right)+(\tilde{H} H)^{-1}(d x+\cos \alpha \omega)^{2}+d s^{2}\left(\mathbb{E}^{3}\right)\right] \\
e^{\phi} & =\left(\tilde{H} H^{-1}\right)^{\frac{3}{4}} \\
F_{2} & =-\sin \alpha d\left(\omega \tilde{H}^{-1}\right)+\tan \alpha d x \wedge d \tilde{H}^{-1},
\end{aligned}
$$

where $d \omega={ }^{*} d H$ and the Hodge duality is with respect to the Euclidean metric in $\mathbb{E}^{3}$. Both the $\left(0_{w} \mid 0\right)_{A}$ and the $\left(0_{m} \mid 6\right)_{A}$ solutions can also be obtained from the 
$\mathrm{D}=11$ plane wave and the KK monopole by first rotating the solutions using (3.7) and (3.8), and then reducing them to $\mathrm{D}=10$ along $u_{11}$. For the $\left(0_{w} \mid 0\right)_{A}$ solution this was done in [18].

Next we turn to the IIA supergravity solutions associated with the bound states $(0 \mid 0,2)_{A}$ and $(0 \mid 0,5)_{A}$. These solutions can be easily derived from the T-duality chains

$$
(2 \mid 2,5)_{M} \stackrel{R}{\rightarrow}(2 \mid 2,4)_{A} \stackrel{T}{\rightarrow}\left(1 \mid 1_{D}, 3\right)_{B} \stackrel{T}{\rightarrow}(0 \mid 0,2)_{A}
$$

and

$$
(2 \mid 2,5)_{M} \stackrel{R}{\rightarrow}(2 \mid 2,5)_{A} \stackrel{T}{\rightarrow}\left(1 \mid 1_{D}, 5_{S}\right)_{B} \stackrel{T}{\rightarrow}(0 \mid 0,5)_{A} .
$$

The $(0 \mid 0,2)_{A}$ and $(0 \mid 0,5)_{A}$ solutions are also obtained by reducing the M-brane solutions along a boost with velocity $v / c=\sin \alpha$ in the direction of compactification, where $c$ is the speed of light (in our units $c=1$ ) )[18]. The appropriate $\mathrm{D}=11$ diffeomorphism which relates the compactifying coordinate $u_{11}$ to the worldvolume time-coordinate $t$ and transverse coordinate $y$ of the M-branes is

$$
u_{11}=\cos ^{-1} \alpha(y+\sin \alpha t), \quad t^{\prime}=\cos ^{-1} \alpha(t+\sin \alpha y)
$$

We also have to 'boost' the harmonic function $H$ (i.e. the mass) of the M-branes as

$$
(1-H) \rightarrow \cos ^{2} \alpha(1-H)
$$

Similarly, reducing the $\mathrm{D}=11$ plane wave along a boost we get the $\left(0 \mid 0_{w}\right)_{A}$ solution (eqn. (3.10)). Finally, boosting the $\mathrm{D}=11 \mathrm{KK}$ monopole, eqn.(3.6), along $\rho$ using (3.15) (setting $y=\rho$ ) and then reducing along $u_{11}$, we get the IIA supergravity solution

$$
\begin{aligned}
d s^{2} & =(\bar{H} \tilde{H})^{\frac{1}{2}}\left[-(\bar{H} \tilde{H})^{-1}(d t+\sin \alpha \cos \alpha \omega)^{2}+\tilde{H}^{-1} d s^{2}\left(\mathbb{E}^{6}\right)+d s^{2}\left(\mathbb{E}^{3}\right)\right] \\
e^{\phi} & =\left(\bar{H} \tilde{H}^{-1}\right)^{\frac{3}{4}} \\
F_{2} & =\sin ^{-1} \alpha d t \wedge d \bar{H}^{-1}-\cos \alpha d\left(\omega \bar{H}^{-1}\right),
\end{aligned}
$$


where

$$
\bar{H}=1+\sin ^{2} \alpha(1-H)
$$

and $d \omega={ }^{*} d H$; the Hodge duality is with respect to the flat metric in $\mathbb{E}^{3}$. We have not been able to derive this solution using a T-duality chain from the $\mathrm{D}=11$ $(2 \mid 2,5)_{M}$ solution as we have done for the rest of the p-brane bound state solutions. Observe that this metric has an off-diagonal term which is reminiscent to a similar term in the Taub-NUT metric. This solution appears to have the appropriate non-vanishing supergravity field strength to be interpreted as a 0-brane/6-brane bound state. However although for $\alpha=0$ we find the familiar 6-brane solution of IIA supergravity, for $\alpha=\frac{\pi}{2}$ we find the 0 -brane solution but with negative ADM mass. In addition, the solution, apart from the singularities at the centres of the harmonic function $H$, has another singularity at $\bar{H}\left(r_{0}\right)=0$ which occurs at small string coupling whenever $\sin \alpha \neq 0$. Note that in order to boost the KK monopole one needs to 'decompactify' the coordinate $\rho$ along the killing direction in order to impose the periodicity in the compactifying $u_{11}$ coordinate. This re-introduces the NUT singularities of the KK monopole that were resolved by the periodic identification of $\rho$. It is not clear though that there is a direct relation between the NUT singularities of the KK monopole and the $\bar{H}\left(r_{0}\right)=0$ ones since in any case they occur at different points. It is more likely that the NUT singularities of the KK monopole are related to the singularities of the $\mathrm{D}=10$ solution that occur at the centres of $H$. The above properties of the solution obscure its interpretation as a 0-brane/6-brane bound state. This appears to be in agreement with the result of [11] that there does not exist such a BPS bound state. 


\section{IIA/IIB T-duality and bound states}

As we have explained in the section 2, the IIA/IIB superstring T-duality and the diffeomorphism invariance of the underlying theories requires the existence of D-p-brane/D- $(\mathrm{p}+2)$-brane bound states in IIA and IIB superstring theories. The D-p-brane bound states present in IIA superstring theory are $(0 \mid 0,2)_{A},(2 \mid 2,4)_{A}$, $(4 \mid 4,6)_{A}$ and $(6 \mid 6,8)_{A}$, and those present in IIB are $\left(1 \mid 1_{D}, 3\right)_{B},\left(3 \mid 3,5_{D}\right)_{B}$ and $\left(5 \mid 5_{D}, 7\right)_{B}$. To find the corresponding IIA supergravity solutions, recall that the $(2 \mid 2,4)_{A}$ solution can be obtained from the $(2 \mid 2,5)_{M}$ solution of M-theory by reducing along one of the fivebrane worldvolume directions orthogonal to the membrane $[16,17]$. Having found $(2 \mid 2,4)_{A}$, we then construct a T-duality chain that extends to the 'left' and the 'right' of this solution as

$$
(0 \mid 0,2)_{A} \stackrel{T}{\rightarrow}\left(1 \mid 1_{D}, 3\right)_{B} \stackrel{T}{\rightarrow}(2 \mid 2,4)_{A} \stackrel{T}{\rightarrow}\left(3 \mid 3,5_{D}\right)_{B} \stackrel{T}{\rightarrow}(4 \mid 4,6)_{A} \stackrel{T}{\rightarrow}\left(5 \mid 5_{D}, 7\right)_{B} \stackrel{T}{\rightarrow}(6 \mid 6,8)_{A},
$$

where the 7-brane is the circularly symmetric one and in last step of this T-duality chain we have used the 'massive' T-duality rules of [28]. The solutions representing the D-p-brane/D-(p+2)-brane bound states can be easily computed from the $(2 \mid 2,4)_{A}$ solution using the chain (4.1). These D-p-brane/D-(p+2)-brane bound states solutions should also be derivable using T-duality transformations at an angle on the IIA and IIB D-(p+1)-branes, i.e. applying a T-duality transformation along the direction $u_{11}$ of (3.7). It turns out that this is the case and the solutions obtained from the two different ways of doing the computation coincide. As an example we derive the IIA $(0 \mid 0,2)_{A}$ bound state solution from the IIB D-1-brane solution. The D-1-brane solution is

$$
\begin{aligned}
d s^{2} & =H^{-\frac{1}{2}} d s^{2}\left(\mathbb{M}^{2}\right)+H^{\frac{1}{2}} d s^{2}\left(\mathbb{E}^{8}\right) \\
F_{3}^{(2)} & =-\epsilon\left(\mathbb{M}^{2}\right) \wedge d H^{-1} \\
e^{\varphi} & =H^{\frac{1}{2}}
\end{aligned}
$$

where $\varphi$ is the IIB dilaton. We parameterise $\mathbb{M}^{2}$ with the coordinates $(t, \rho)$, and $\mathbb{E}^{8}$ with the coordinates $\left\{y^{1}, \ldots, y^{8}\right\}$ and set $y=y^{1}$. Then we change coordinates from 
$(\rho, y)$ to $\left(u_{10}, u_{11}\right)$ as in $(3.7)$ and $(3.8)$, and perform a T-duality transformation along $u_{11}$. The resulting IIA $(0 \mid 0,2)_{A}$ solution is

$$
\begin{aligned}
d s^{2} & =-H^{-\frac{1}{2}} d t^{2}+H^{\frac{1}{2}} \tilde{H}^{-1} d s^{2}\left(\mathbb{E}^{2}\right)+H^{\frac{1}{2}} d s^{2}\left(\mathbb{E}^{7}\right) \\
e^{\phi} & =H^{\frac{3}{4}} \tilde{H}^{-\frac{1}{2}} \\
F_{2} & =-\sin \alpha d t \wedge d H^{-1} \\
F_{4} & =\cos \alpha \epsilon\left(\mathbb{M}^{3}\right) \wedge d H^{-1} \\
F_{3} & =\tan \alpha \epsilon\left(\mathbb{E}^{2}\right) \wedge d \tilde{H}^{-1}
\end{aligned}
$$

in agreement with [18], where $\phi$ is the IIA dilaton. Similarly, the bound states $\left(0_{w} \mid 1_{F}\right)$ and $\left(0_{m} \mid 5_{S}\right)$ expected from applying T-duality at an angle to the (IIA or IIB) fundamental string, the solitonic 5-brane, the plane wave and the KK monopole are as follows: The $\left(0_{w} \mid 1_{F}\right)$ solution is

$$
\begin{aligned}
d s^{2} & =-H^{-1} d t^{2}+H \tilde{H}^{-1}\left(d x+\sin \alpha(H-1) H^{-1} d t\right)^{2}+\tilde{H}^{-1} d z^{2}+d s^{2}\left(\mathbb{E}^{7}\right) \\
e^{\phi} & =\tilde{H}^{-\frac{1}{2}} \\
F_{3} & =-\cos ^{-1} \alpha d t \wedge d z \wedge d \tilde{H}^{-1}-\tan \alpha d x \wedge d z \wedge d \tilde{H}^{-1}
\end{aligned}
$$

and it can be interpreted as a boosted fundamental string so it is diffeomorphic to the fundamental string solution [29] (up to a rescalling of $H-1$ ). The $\left(0_{m} \mid 5_{S}\right.$ ) solution is

$$
\begin{aligned}
d s^{2} & =d s^{2}\left(\mathbb{M}^{5}\right)+H \tilde{H}^{-1} d z^{2}+\tilde{H}^{-1}(d x+\cos \alpha \omega)^{2}+H d s^{2}\left(\mathbb{E}^{3}\right) \\
e^{\phi} & =H^{\frac{1}{2}} \tilde{H}^{-\frac{1}{2}} \\
F_{3} & =-\sin \alpha d z \wedge d\left(\omega \tilde{H}^{-1}\right)-\tan \alpha d x \wedge d z \wedge d \tilde{H}^{-1},
\end{aligned}
$$

where $\omega$ is as in (3.17).

Next we turn to examine the solutions corresponding to the p-brane bound states which are expected from applying a T-duality along a boost to the (IIA or IIB) D-p-branes. As we have explained in section 2, these bound states always 
involve the (IIA or IIB) fundamental string. The corresponding solutions can be found from the T-duality chains

$$
(2 \mid 2,5)_{M} \stackrel{R}{\rightarrow}\left(1 \mid 1_{F}, 4\right)_{A} \stackrel{T}{\rightarrow}\left(1 \mid 1_{F}, 3\right)_{B} \stackrel{T}{\rightarrow}\left(1 \mid 1_{F}, 2\right)_{A} \stackrel{T}{\rightarrow}\left(1 \mid 1_{F}, 1_{D}\right)_{B}
$$

and

$$
(2 \mid 2,5)_{M} \stackrel{R}{\rightarrow}\left(1 \mid 1_{F}, 4\right)_{A} \stackrel{T}{\rightarrow}\left(1 \mid 1_{F}, 5_{D}\right)_{B} \stackrel{T}{\rightarrow}\left(1 \mid 1_{F}, 6\right)_{A} \stackrel{T}{\rightarrow}\left(1 \mid 1_{F}, 7\right)_{B} \stackrel{T}{\rightarrow}\left(1 \mid 1_{F}, 8\right)_{A} .
$$

It turns out that these bound states can also be derived by first using the $D=10$ diffeomorphism (3.15) together with (3.16), and then performing a T-duality transformation along $u_{11}$. Thus confirming our interpretation in section 2 for the origin of these bound states. We shall not present all these solutions here since they can be easily derived by applying the T-duality rules [21]. In any case, some of these solutions are already known like for example the $\left(1 \mid 1_{F}, 4\right)_{A}$ and the $\left(1 \mid 1_{F}, 1_{D}\right)_{B}$ solutions; the latter is associated with the $\operatorname{IIB}(p, q)$ strings $[19,20]$. Nevertheless, we present the $\left(1 \mid 1_{F}, 6\right)_{A}$ solution

$$
\begin{aligned}
d s^{2} & =\tilde{H}^{\frac{1}{2}}\left[H^{-1} d s^{2}\left(\mathbb{M}^{2}\right)+\tilde{H}^{-1} d s^{2}\left(\mathbb{E}^{5}\right)+d s^{2}\left(\mathbb{E}^{3}\right)\right] \\
e^{\phi} & =\tilde{H}^{-\frac{1}{4}} H^{-\frac{1}{2}} \\
F_{3} & =-\sin \alpha \epsilon\left(\mathbb{M}^{2}\right) \wedge d H^{-1}+\sin \alpha \cos \alpha d t \wedge d\left(H^{-1} \omega\right) \\
F_{2} & =\cos \alpha \star d H
\end{aligned}
$$

as another example, where $\omega$ is given as in (3.17). Next applying T-duality along a boost to the (IIA or IIB) fundamental string, we do not find a new bound state. To be more precise, the $\mathrm{D}=10$ solution that one finds can be brought into the form of that of the (IIB or IIA) fundamental string up to a $\mathrm{D}=10$ diffeomorphism. Similarly, applying T-duality along a boost to the plane wave we get the $\left(0_{w} \mid 1_{F}\right)$ solution as in eqn. (4.5). The $\left(0_{m} \mid 1_{F}\right)$ bound state solution associated with a 
boosted solitonic (IIA or IIB) 5-brane is

$$
\begin{aligned}
d s^{2} & =-\tilde{H} H^{-1} d t^{2}+d s^{2}\left(\mathbb{E}^{5}\right)+H^{-1}(d x+\cos \alpha \omega)^{2}+\tilde{H} d s^{2}\left(\mathbb{E}^{3}\right) \\
F_{3} & =\sin \alpha d t \wedge d x \wedge d H^{-1}-\cos \alpha \sin \alpha d t \wedge d\left(\omega H^{-1}\right) \\
e^{\phi} & =\tilde{H}^{\frac{1}{2}} H^{-\frac{1}{2}}
\end{aligned}
$$

where $\omega$ is as in (3.17). Finally, applying T-duality along a boost to the KK monopole yields the $\left(1 \mid 1_{F}, 5_{S}\right)$ solution

$$
\begin{aligned}
d s^{2} & =-\bar{H}^{-1}(d t+\sin \alpha \cos \alpha \omega)^{2}+d s^{2}\left(\mathbb{E}^{5}\right)+\bar{H}^{-1} \tilde{H} d x^{2}+\tilde{H} d s^{2}\left(\mathbb{E}^{3}\right) \\
e^{\phi} & =\left(\tilde{H} \bar{H}^{-1}\right)^{\frac{1}{2}} \\
F_{3} & =\sin ^{-1} \alpha d t \wedge d x \wedge d \bar{H}^{-1}+\cos \alpha d x \wedge d\left(\omega \bar{H}^{-1}\right)
\end{aligned}
$$

where $\bar{H}$ and $\omega$ is given as in (3.17). The interpretation of this solution as a fundamental string/solitonic 5-brane bound state is obscured for similar reasons to that of the interpretation of the solution (3.17) as a 0-brane/6-brane bound state. We shall not repeat the argument here.

\section{M-theory and p-brane bound states}

Some of the bound state solutions of IIA supergravity derived in the previous two sections can be lifted to eleven dimensions to yield new BPS solutions of $\mathrm{D}=11$ supergravity preserving $1 / 2$ of the spacetime supersymmetry. The bound states that we shall lift here are $\left(0_{m} \mid 5_{S}\right),(4 \mid 4,6)_{A},(1 \mid 1,6)_{A}$ and $\left(0_{m} \mid 1_{F}\right)$. The $\mathrm{D}=11$ solution that yields $\left(0_{m} \mid 5_{S}\right)$ or $(4 \mid 4,6)_{A}$ after reduction has the interpretation of a $\mathrm{D}=11$ fivebrane/KK monopole bound state and it can be expressed as

$$
\begin{aligned}
d s^{2}= & H^{\frac{2}{3}} \tilde{H}^{\frac{1}{3}}\left[H^{-1} d s^{2}\left(\mathbb{M}^{5}\right)+\tilde{H}^{-1} d s^{2}\left(\mathbb{E}^{2}\right)\right. \\
& \left.+(H \tilde{H})^{-1}(d x+\cos \alpha \omega)^{2}+d s^{2}\left(\mathbb{E}^{3}\right)\right] \\
G_{4}= & -\sin \alpha \epsilon\left(\mathbb{E}^{2}\right) \wedge d\left(\omega \tilde{H}^{-1}\right)-\tan \alpha \epsilon\left(\mathbb{E}^{2}\right) \wedge d x \wedge d \tilde{H}^{-1},
\end{aligned}
$$

where $\omega$ is as in (3.17). Finally the $\mathrm{D}=11$ solution that yields $(1 \mid 1,6)_{A}$ or $\left(0_{m} \mid 1_{F}\right)$ after reduction has the interpretation of a $\mathrm{D}=11$ membrane/KK monopole bound 
state and it can be expressed as

$$
\begin{aligned}
d s^{2}= & \tilde{H}^{\frac{2}{3}} H^{\frac{1}{3}}\left[-H^{-1} d t^{2}+\tilde{H}^{-1} d s^{2}\left(\mathbb{E}^{5}\right)+H^{-1} d z^{2}\right. \\
& \left.+(H \tilde{H})^{-1}(d x+\cos \alpha \omega)^{2}+d s^{2}\left(\mathbb{E}^{3}\right)\right] \\
G_{4}= & \sin \alpha \cos \alpha d t \wedge d z \wedge d\left(\omega H^{-1}\right)+\sin \alpha d t \wedge d z \wedge d x \wedge d H^{-1} .
\end{aligned}
$$

\section{Concluding remarks}

It is well known that apart from the M-brane solutions of $\mathrm{D}=11$ supergravity, there are other solutions that have the interpretation of intersecting M-branes [30]. The standard reduction of these configurations $[30,31,16,32,33,34]$ to $\mathrm{D}=10$ has been extensively studied yielding solutions in $\mathrm{D}=10$ with the interpretation of intersecting IIA p-branes. It is clear that one can reduce the intersecting Mbrane solutions along a generic $\mathrm{D}=11$ direction $u$. There are many ways to choose such a $\mathrm{D}=11$ direction. For this, let us consider the intersecting M-brane solution $\left(k \mid p_{1}, \ldots, p_{\ell}\right)$. Then $u$ can be chosen as a linear combination of (i) a k-brane spatial worldvolume direction and a 'relative transverse' direction of the configuration, (ii) a k-brane spatial worldvolume direction and a 'overall transverse' direction of the configuration, (iii) two 'relative transverse' directions of the configuration with the metric having different components along these directions and (iv) a 'relative transverse' and a 'overall transverse' directions of the configuration. Each such choice will lead to a different $D=10$ reduction of the $D=11$ solution. We can also boost the intersecting M-brane solutions in different ways and then reduce them along the direction of the boost thus producing many more new bound states in $\mathrm{D}=10$. The same applies for the T-duality transformations acting on the IIA and IIB intersecting brane configurations. Thus many more solutions can be constructed in $\mathrm{D}=10$ by chains of T-duality transformations all preserving the same amount of spacetime supersymmetry as the original solution. Most of these new solutions have the interpretation of bound states of intersecting p-branes or that of intersecting p-brane bound states [35]. 
The argument that we have used to propose the existence of p-brane bound states in IIA and IIB superstring theories also applies in $D<10$. Apart from the p-brane bound states that are obtained by reducing the $\mathrm{D}=10 \mathrm{p}$-brane bound states that we have found in section 2 to lower dimensions, there should also exist additional p-brane bound states in $D<10$. This will be the case whenever a duality between two superstring theories breaks some of their reparameterisation invariance.

Note added in the proof

During the preparation of this paper we have received [36] in which the solutions interpreted as D-p-brane/D-(p+2)-brane bound states are constructed from the Dbrane solutions using rotations and T-duality transformations. This paper overlaps with some of our material in section 4 .

Acknowledgments: We would like to thank M. Douglas, G.W. Gibbons, M.B. Green, C.M. Hull, M. Perry and P.K. Townsend for helpful comments. G.P. is supported by a University Research Fellowship from the Royal Society. M.S.C. is funded by JNICT (Portugal) under the programme PRAXIS XXI.

\section{REFERENCES}

1. P.K. Townsend, Phys. Lett. 350B (1995) 184.

2. E. Witten, Nucl. Phys. B443 (1995) 85.

3. G.T. Horowitz and A. Strominger, Nucl. Phys. B360 (1991) 197.

4. M. J. Duff and J. X. Lu, Nucl. Phys. B416(1994) 301.

5. J. Polchinski, Phys. Rev. Lett. 75 (1995) 4724.

6. M. Douglas, D. Kabat, P. Pouliot and S.H. Shenker, Nucl. Phys. B485 (1997) 85, hep-th/9608024. 
7. G. Papadopoulos and P.K. Townsend, Phys. Lett. B393 (1997) 59, hepth/9609095.

8. M. Dine, P. Huet and N. Seiberg, Nucl. Phys. B322 (1989) 301.

9. J. Dai, R. Leigh and J. Polchinski, Mod. Phys. Lett. A4 (1989) 2073.

10. M. Douglas, Branes within Branes, hep-th/9512077.

11. J. Polchinski, TASI Lectures on D-branes, hep-th/9611050.

12. M.B. Green and M. Gutperle, Nucl. Phys. B476 (1996) 484.

13. A. Sen, Phys. Rev. D54 (1996) 2964.

14. G. Lifschytz, Probing bound states of D-branes, hep-th/9610125.

15. J.M. Izquierdo, N.D. Lambert and G. Papadopoulos and P.K. Townsend, Nucl. Phys. B460, (1996) 560.

16. G. Papadopoulos, A brief guide to p-branes, hep-th/9604068.

17. M.B. Green, N.D. Lambert, G. Papadopoulos and P.K. Townsend, Phys. Lett. 384B (1996) 86.

18. J. Russo and A. Tseytlin, Nucl. Phys. B490 (1997) 121, hep-th/9611047.

19. J.H. Schwarz, Phys. Lett.B360 (1995) 13 (E: B364 (1995) 252); Phys. Lett. B367 (1996) 97.

20. E. Witten, Nucl. Phys. B460 (1995) 335.

21. E. Bergshoeff, C.M. Hull and T. Ortín, Nucl. Phys. B451 (1995) 547.

22. P.K. Townsend, Phys. Lett. 227B (1992) 285; Four Lectures on M-theory, hep-th/9612121.

E. Bergshoeff, L.A.J. London and P.K. Townsend, Class. Quantum Grav. 9 (1992) 2545.

23. M J Duff and K S Stelle Phys. Lett. B253(1991) 113.

24. R. Güven, Phys. Lett. 276B (1992) 49. 
25. G.W. Gibbons and S.W. Hawking, Phys. Lett. 78B (1978) 430.

26. G.W. Gibbons, Nucl. Phys. B207 (1982) 337.

27. C.M. Hull, Phys. Lett. B139 (1984) 39.

28. E. Bergshoeff, M. de Roo, M.B. Green, G. Papadopoulos and P.K. Townsend, Nucl. Phys. B470 (1996) 113.

29. A. Dabholkar, G.W. Gibbons, J. Harvey and F. Ruiz Ruiz, Nucl. Phys. B340 (1990) 33; A. Dabholkar and J. Harvey, Phys. Rev. Lett. 63 (1989) 478.

30. G. Papadopoulos and P.K. Townsend, Phys. Lett. 380B (1996) 273.

31. A. Tseytlin, Nucl. Phys. B475 (1996) 149.

32. I. Klebanov and A. Tseytlin, Nucl. Phys. B475 (1996) 179.

33. J.P. Gauntlett, D.A. Kastor and J. Traschen, Nucl. Phys. B478 (1996) 544.

34. K. Behrndt, E. Bergshoeff and B. Janssen, Phys. Rev. D55 (1997) 3785, hep-th/9604168.

35. M.S. Costa, Nucl. Phys. B490 (1997) 202, hep-th/9609181.

36. J.C. Breckenridge, G. Michaud and R.C. Myers, Phys. Rev. D55 (1997) 6438, hep-th/9611174. 